\title{
Effektiv bei hypoxämischer Atmungsinsuffizienz
}

Fragestellung: Ist die High-flow-Sauerstofftherapie genau so effektiv wie die nicht invasive Ventilation bei (postoperativen) $\mathrm{Pa}$ tienten mit Hypoxämie?

Hintergrund: Die nicht invasive Ventilationstherapie (NIV) kann die Reintubationsrate und die Mortalität nach kardiochirurgischen Eingriffen senken [1, 2]. Der Einsatz von NIV ist personal- und zeitintensiv und wird von den Patienten nicht immer gut toleriert.

Die High-flow-Sauerstofftherapie wird zunehmend angewandt aufgrund der Einfachheit der Applikation und der besseren Patiententoleranz. Es ist unklar, ob diese Therapie eine Alternative zur NIV darstellen kann.

Originalie

Stéphan F, Barrucand B, Petit P et al. High-Flow Nasal Oxygen vs Noninvasive Positive Airway Pressure in Hypoxemic Patients After Cardiothoracic Surgery: A Randomized Clinical Trial. JAMA 2015; 313(23):2331-9.
Material und Methoden: In dieser multizentrischen, prospektiv-randomisierten Studie wurden 830 Patienten nach einem kardiochirurgischen Eingriff eingeschlossen. Es wurden Patienten mit einer Hypoxämie $\left(\mathrm{SpO}_{2}\right.$ $<90 \%)$ trotz konventioneller Sauerstoffgabe (12 Liter/min oder Sauerstoffzumischung von 50\%) und Patienten mit Risikofaktoren für ein respiratorisches Versagen $(\mathrm{BMI}>30$, eingeschränkte linksventrikuläre Funktion) eingeschlossen. Die eine Gruppe erhielt High-flowSauerstofftherapie $\left(\mathrm{FiO}_{2} 50 \%\right.$ bei einem Fluss von $\left.50 \mathrm{Liter} / \mathrm{min}\right)$ und die andere Gruppe NIV (PEEP $4 \mathrm{~cm} \mathrm{H}_{2} \mathrm{O}$, Druckunterstützung $8 \mathrm{~cm} \mathrm{H} \mathrm{H}_{2} \mathrm{O}$ mit einem Ziel-Tidalvolumen von $8 \mathrm{ml} / \mathrm{kg} \mathrm{KG}$, ca. 6 Stunden pro Tag).

Ergebnisse: Das primäre Endziel „Therapieversagen“, definiert als Reintubation, Abbruch oder Wechsel der Therapie war in beiden Gruppen gleich (21\% in der High-flow-Gruppe vs. 20,9\% in der NIV-Gruppe). Die Mortalität unterschied sich ebenfalls nicht signifikant (high flow: 6,8\%, NIV: 5,5\%). Der Einsatz beider Verfahren führte zu einer Verbesserung des $\mathrm{PaO}_{2}$ :FiO - -Verhältnisses über die Zeit. NIV war assoziiert mit einer besseren Oxygenierung $\left(\mathrm{PaO}_{2}: \mathrm{FiO}_{2}\right.$-Verhältnis) bei höherer Atemfrequenz. Beide Gruppen waren normokapnisch. Dyspnoe und Patientenkomfort waren ähnlich in beiden Gruppen, während der pflegerische Aufwand bei NIV höher war.

Schlussfolgerung: Bei hypoxämischer Atmungsinsuffizienz nach kardiochirurgischen Eingriffen kann die High-flow-Sauerstofftherapie mit einer ähnlichen Sicherheit wie die NIV eingesetzt werden.

\section{Zwischen hypoxämischer und hyperkapnischer Atmungsinsuffizienz unterscheiden!}

Die High-flow-Sauerstofftherapie ist eine effektive und sichere Therapie bei spontan atmenden Patienten mit hypoxämischer Atmungsinsuffizienz. Die positiven Effekte beruhen auf der höheren applizierte Sauerstoffkonzentration, auf einer guten mukoziliären Clearance durch die Anwärmung [3] und auf dem Aufbau eines positiven Atemwegsdruckes [4] bei gleichzeitiger Senkung des Totraums durch den hohen Gasfluss, was wiederum die Atemfrequenz und die Atemarbeit senken kann.

Es sollte allerdings zwischen hypoxämischer und hyperkapnischer Atmungsinsuffizienz unterschieden werden. Während bei der hypoxämischen Atmungsinsuffizienz die High-flowSauerstofftherapie der NIV mindestens gleichwertig ist, ist das bei der hyperkapnischen Atmungsinsuffizienz nicht der Fall. Hier bleibt nach der aktuellen Datenlage (aber auch aufgrund pathophysiologischer Überlegungen) die NIV weiterhin die Therapie der Wahl.

\section{Literatur}

1. Guarracino F, Cabrini L, Ferro B, et al. Noninvasive ventilation practice in cardiac surgery patients: insights from a European survey. Journal of cardiothoracic and vascular anesthesia. Oct 2013;27(5):e63-65.
2. Nava S, Hill N. Non-invasive ventilation in acute respiratory failure. Lancet. Jul 18 2009;374(9685):250-9.

3. Hasani A, Chapman TH, McCool D, Smith RE, Dilworth JP, Agnew JE. Domiciliary humidification improves lung mucociliary clearance in patients with bronchiectasis. Chronic respiratory disease. 2008;5(2):81-6.

4. Groves N, Tobin A. High flow nasal oxygen generates positive airway pressure in adult volunteers. Australian critical care : official journal of the Confederation of Australian Critical Care Nurses. Nov 2007;20(4):126-31.

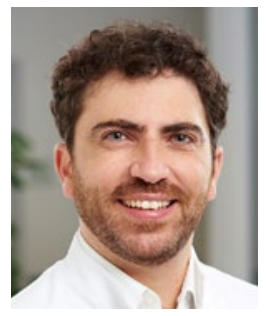

Georgios Sofianos

Facharzt für Innere Medizin, Kardiologie und Intensivmedizin

Krankenhaus Bethanien gGmbH

Aufderhöher Straße 169-175

42699 Solingen

georgios.sofianos@klinik-bethanien.de 